\title{
Gas kinematics and star formation in the filamentary molecular cloud G47.06+0.26*
}

\author{
Jin-Long $\mathrm{Xu}^{1,5}$, Ye $\mathrm{Xu}^{2,6}$, Chuan-Peng Zhang ${ }^{1,5}$, Xiao-Lan Liu ${ }^{1,5}$, Naiping $\mathrm{Yu}^{1,5}$, \\ Chang-Chun Ning ${ }^{3,5}$, and Bing-Gang Ju ${ }^{4,6}$ \\ 1 National Astronomical Observatories, Chinese Academy of Sciences, Beijing 100012, PR China \\ e-mail: xuj1@bao.ac.cn \\ 2 Purple Mountain Observatory, Chinese Academy of Sciences, Nanjing 210008, PR China \\ 3 Tibet University, Lhasa, Tibet 850000, PR China \\ ${ }^{4}$ Purple Mountain Observatory, Qinghai Station, Delingha 817000, PR China \\ 5 NAOC-TU Joint Center for Astrophysics, Lhasa 850000, PR China \\ ${ }^{6}$ Key Laboratory of Radio Astronomy, Chinese Academy of Sciences, PR China
}

Received 27 June 2016 / Accepted 21 August 2017

\begin{abstract}
Aims. We performed a multi-wavelength study toward the filamentary cloud G47.06+0.26 to investigate the gas kinematics and star formation.

Methods. We present the ${ }^{12} \mathrm{CO}(J=1-0),{ }^{13} \mathrm{CO}(J=1-0)$ and $\mathrm{C}^{18} \mathrm{O}(J=1-0)$ observations of $\mathrm{G} 47.06+0.26$ obtained with the Purple Mountain Observation (PMO) 13.7 m radio telescope to investigate the detailed kinematics of the filament. Radio continuum and infrared archival data were obtained from the NRAO VLA Sky Survey (NVSS), the APEX Telescope Large Area Survey of the Galaxy (ATLASGAL), the Galactic Legacy Infrared Mid-Plane Survey Extraordinaire (GLIMPSE) survey, and the Multi-band Imaging Photometer Survey of the Galaxy (MIPSGAL). To trace massive clumps and extract young stellar objects in G47.06+0.26, we used the BGPS catalog v2.0 and the GLIMPSE I catalog, respectively.

Results. The ${ }^{12} \mathrm{CO}(J=1-0)$ and ${ }^{13} \mathrm{CO}(J=1-0)$ emission of $\mathrm{G} 47.06+0.26$ appear to show a filamentary structure. The filament extends about $45^{\prime}(58.1 \mathrm{pc})$ along the east-west direction. The mean width is about $6.8 \mathrm{pc}$, as traced by the ${ }^{13} \mathrm{CO}(J=1-0)$ emission. G47.06+0.26 has a linear mass density of $\sim 361.5 M_{\odot} \mathrm{pc}^{-1}$. The external pressure (due to neighboring bubbles and H II regions) may help preventing the filament from dispersing under the effects of turbulence. From the velocity-field map, we discern a velocity gradient perpendicular to G47.06+0.26. From the Bolocam Galactic Plane Survey (BGPS) catalog, we found nine BGPS sources in G47.06+0.26, that appear to these sources have sufficient mass to form massive stars. We obtained that the clump formation efficiency (CFE) is $\sim 18 \%$ in the filament. Four infrared bubbles were found to be located in, and adjacent to, G47.06+0.26. Particularly, infrared bubble N98 shows a cometary structure. CO molecular gas adjacent to N98 also shows a very intense emission. H II regions associated with infrared bubbles can inject the energy to surrounding gas. We calculated the kinetic energy, ionization energy, and thermal energy of two H II regions in G47.06+0.26. From the GLIMPSE I catalog, we selected some Class I sources with an age of $\sim 10^{5} \mathrm{yr}$, which are clustered along the filament. The feedback from the H II regions may cause the formation of a new generation of stars in filament G47.06+0.26.
\end{abstract}

Key words. stars: formation - stars: early-type - ISM: individual objects: G47.06+0.26 - ISM: individual objects: N98 - HII regions

\section{Introduction}

Massive stars have a significant effect on the Galactic environment, but their formation is not well understood. It is known that $70-90 \%$ of stars are born in clusters in giant molecular clouds (GMCs; Lada \& Lada 2003), which usually show filamentary structures (Busquet et al. 2013). Filaments have recently attracted a lot of attention, especially as some dust continuum Galactic plane surveys have revealed a wealth of filamentary structures in star-forming clouds, such as the APEX Telescope Large Area Survey of the Galaxy (ATLASGAL; Schuller et al. 2009) and the Herschel infrared Galactic Plane Survey (Hi-GAL; Molinari et al. 2010). Massive stars predominantly form in the most bound parts of filaments (André et al. 2014), while low-mass protostars tend to form in less-bound sites (Foster et al. 2014; Xu et al. 2016).

* The reduced datacubes (FITS files) are only available at the CDS via anonymous ftp to cdsarc.u-strasbg. fr (130.79.128.5) or via http://cdsarc.u-strasbg.fr/viz-bin/qcat?]/A+A/609/A43
Theoretical works show that filamentary molecular clouds can fragment into clumps due to gravitational instabilities (Inutsuka \& Miyama 1992; Nakamura et al. 1993; Tomisaka 1995). These clumps are not randomly distributed in the filamentary molecular cloud, but along filaments, like beads on a string. Once the clumps are formed, some of them will evolve, collapse, and form stars. Contreras et al. (2016) found a total of 101 clumps within five filaments using the ATLASGAL $870 \mu \mathrm{m}$ continuum data. They found that most clumps have sufficient mass and density to form massive stars. Moreover, the filaments can also enhance accretion rates of individual star-forming cores by material flowing from the filament to the clumps (Banerjee \& Pudritz 2008; Myers 2009). Hence, filamentary molecular clouds are usually associated with massive star-forming regions. Once a massive star forms inside a filament, UV radiation and stellar winds will ionize the surrounding gas and create an infrared bubble (Churchwell et al. 2006; Zhang et al. 2015), and perhaps even disrupt the natal molecular cloud (Jackson et al. 2010). In addition, the expanding 
Table 1. The different surveys.

\begin{tabular}{lcc}
\hline \hline Survey & Waveband & Reference \\
\hline NRAO VLA Sky & $21 \mathrm{~cm}$ & Condon et al. (1998) \\
BGPS & $1.1 \mathrm{~mm}$ & Ginsburg et al. (2013) \\
ATLASGAL & $870 \mu \mathrm{m}$ & Schuller et al. (2009) \\
GLIMPSE & $3.6,4.5,5.8,8.0 \mu \mathrm{m}$ & Benjamin et al. (2003) \\
MIPSGAL & $24 \mu \mathrm{m}$ & Carey et al. (2009) \\
\hline
\end{tabular}

$\mathrm{H}$ II region and stellar winds will entrain and accelerate ambient gas and inject momentum and energy into the surrounding environment (Narayanan et al. 2008; Arce et al. 2011). H II regions contain kinetic energy, ionization energy and thermal energy. Hence, feedback from young massive stars has been proposed as a significant aspect of the self-regulation of star formation (e.g., Norman \& Silk 2008; Franco 1983; Liu et al. 2016; Dewangan et al. 2016). Particularly, if feedback can maintain the observed turbulence in molecular clouds, then it can be responsible for stabilizing the clouds against gravitational collapse (Shu et al. 1987).

GMC G47.06+0.26, with a total mass of $2.0 \times 10^{4} M_{\odot}$ and a distance of $4.44 \mathrm{kpc}$, shows a filamentary structure (Wang et al. 2015). G47.06+0.26, as a long, dense GMC, is a good laboratory to investigate the connection between filaments and star formation, and to understand the processes that lead to the fragmentation of a filament.

In this paper, we present the results of a multi-wavelength study to investigate the gas kinematics and star formation in filament G47.06+0.26. Combining our data with those obtained by the NRAO VLA Sky survey, the ATLASGAL survey, and the GLIMPSE survey, our aim was to construct a comprehensive large-scale picture of G47.06+0.26. Our observations and data reduction are described in Sect. 2, and the results are presented in Sect. 3. In Sect. 4, we discuss the star-formation scenario, while our conclusions are summarized in Sect. 5.

\section{Observations and data reduction}

The mapping observations of filament G47.06+0.26 were performed in the ${ }^{12} \mathrm{CO}(J=1-0),{ }^{13} \mathrm{CO}(J=1-0)$, and $\mathrm{C}^{18} \mathrm{O}$ $(J=1-0)$ lines using the Purple Mountain Observation (PMO) $13.7 \mathrm{~m}$ radio telescope at De Ling $\mathrm{Ha}$ in western China at an altitude of $3200 \mathrm{~m}$, during October 2015 . The $3 \times 3$ beam array receiver system in single-sideband (SSB) mode was used as front end. The back end is a fast Fourier transform spectrometer (FFTS) of 16384 channels with a bandwidth of $1 \mathrm{GHz}$. ${ }^{12} \mathrm{CO} J=1-0$ was observed at upper sideband with a system noise temperature (Tsys) of $270 \mathrm{~K}$, while ${ }^{13} \mathrm{CO} J=1-0$ and $\mathrm{C}^{18} \mathrm{O} J=1-0$ were observed simultaneously at lower sideband with a system noise temperature of $146 \mathrm{~K}$. The half-power beam width (HPBW) was 53" at $115 \mathrm{GHz}$. The total mapping area is $30^{\prime} \times 20^{\prime}$ in ${ }^{12} \mathrm{CO}(J=1-0),{ }^{13} \mathrm{CO}(J=1-0)$, and $\mathrm{C}^{18} \mathrm{O}(J=1-0)$ with a $0.5^{\prime} \times 0.5^{\prime}$ grid. $\mathrm{Xu}$ et al. (2016) gave more details of the PMO $13.7 \mathrm{~m}$ radio telescope instrumentation.

In addition, we also used survey data from different wavebands, which are listed in Table 1.

\section{Results}

\subsection{Carbon monoxide, infrared, and radio continuum emission of $\mathrm{G} 47.06+0.26$}

Figure 1 shows the averaged spectra of ${ }^{12} \mathrm{CO}(J=1-0)$, ${ }^{13} \mathrm{CO}(J=1-0)$, and $\mathrm{C}^{18} \mathrm{O}(J=1-0)$ over the filamentary

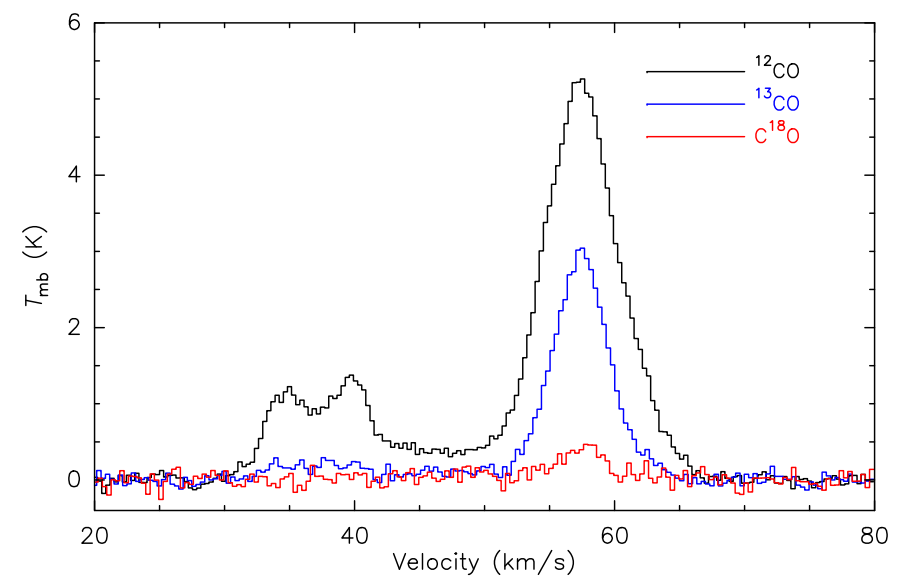

Fig. 1. Averaged spectra of ${ }^{12} \mathrm{CO}(J=1-0),{ }^{13} \mathrm{CO}(J=1-0)$, and $\mathrm{C}^{18} \mathrm{O}(J=1-0)$ over the entire filament. We note that the intensities of ${ }^{13} \mathrm{CO}(J=1-0)$ and $\mathrm{C}^{18} \mathrm{O}(J=1-0)$ are multiplied by factors of two and three for clarity, respectively.

molecular cloud G47.06+0.26. We find that there are two main components at velocity intervals $30 \sim 50 \mathrm{~km} \mathrm{~s}^{-1}$ and $50 \sim 67 \mathrm{~km} \mathrm{~s}^{-1}$, respectively. The LSR velocity of G47.06+0.26 is $57.5 \mathrm{~km} \mathrm{~s}^{-1}$ in ${ }^{13} \mathrm{CO}(J=1-0)$ (Wang et al. 2015), then the $\mathrm{CO}$ emission component in the velocity interval of $50 \sim 67 \mathrm{~km} \mathrm{~s}^{-1}$ is associated with $\mathrm{G} 47.06+0.26$. The $\mathrm{C}^{18} \mathrm{O}(J=1-0)$ emission of $\mathrm{G} 47.06+0.26$ is very weak $(0.13 \mathrm{~K})$. The ${ }^{12} \mathrm{CO}(J=1-0)$ emission shows another component at $30 \sim 50 \mathrm{~km} \mathrm{~s}^{-1}$, originating from independent material along the line of sight. Using the Hi-GAL data, Wang et al. (2015) found that the dust continuum emission of G47.06+0.26 shows a bent C-shape. To find any associated components with G47.06+0.26, we made channel maps in ${ }^{13} \mathrm{CO}(J=1-0)$. Through the channel maps, we obtain that the ${ }^{13} \mathrm{CO}(J=1-0)$ emission detected in the velocity range between 52 and $64 \mathrm{~km} \mathrm{~s}^{-1}$ are well correlated with the dust continuum emission of G47.06+0.26. The velocity component in the interval $30 \sim 50 \mathrm{~km} \mathrm{~s}^{-1}$ is likely foreground or background emission not related with $\mathrm{G} 47.06+0.26$.

Using the velocity range of 52 to $64 \mathrm{~km} \mathrm{~s}^{-1}$, we made integrated intensity maps of ${ }^{12} \mathrm{CO}(J=1-0),{ }^{13} \mathrm{CO}(J=1-0)$, and $\mathrm{C}^{18} \mathrm{O}(J=1-0)$, as shown in Fig. 2. From a large-scale perspective, the ${ }^{12} \mathrm{CO}(J=1-0)$ and ${ }^{13} \mathrm{CO}(J=1-0)$ emission of G47.06+0.26 shows a filamentary structure extending from west to east. The region traced by ${ }^{12} \mathrm{CO}(J=1-0)$ is more diffuse than that of ${ }^{13} \mathrm{CO}(J=1-0)$. We divided the filament into region I and region II, and found that $\mathrm{C}^{18} \mathrm{O}(J=1-0)$ is only seen in region I, indicating that it is denser than in region II. Several clumps are found in the filament. Adopting a distance of $4.44 \mathrm{kpc}$ (Wang et al. 2015), the filament extends about $58.1 \mathrm{pc}$ $\left(45.0^{\prime}\right)$ in length, while the mean width is about $6.8 \mathrm{pc}\left(5.3^{\prime}\right)$, as traced by the ${ }^{13} \mathrm{CO}(J=1-0)$ emission. The aspect ratio is $9: 1$. The measured length is a lower limit, due to the unknown inclination.

Figures 3a,b display the ${ }^{13} \mathrm{CO}(J=1-0)$ emission maps overlaid on the Spitzer-IRAC $8 \mu \mathrm{m}$ and Spitzer-MIPSGAL $24 \mu \mathrm{m}$ emission of $\mathrm{G} 47.06+0.26$, respectively. We find some bright $8 \mu \mathrm{m}$ emission along, and adjacent to, the filamentary molecular cloud, whose image is visually similar to the $24 \mu \mathrm{m}$ emission. Generally, the bright Spitzer-IRAC $8 \mu \mathrm{m}$ emission is attributed to polycyclic aromatic hydrocarbons (PAHs; Leger \& Puget 1984). The PAH molecules are excited in the photodissociation regions (PDRs) by UV radiation arising from $\mathrm{H}$ II regions, however, they can also be 

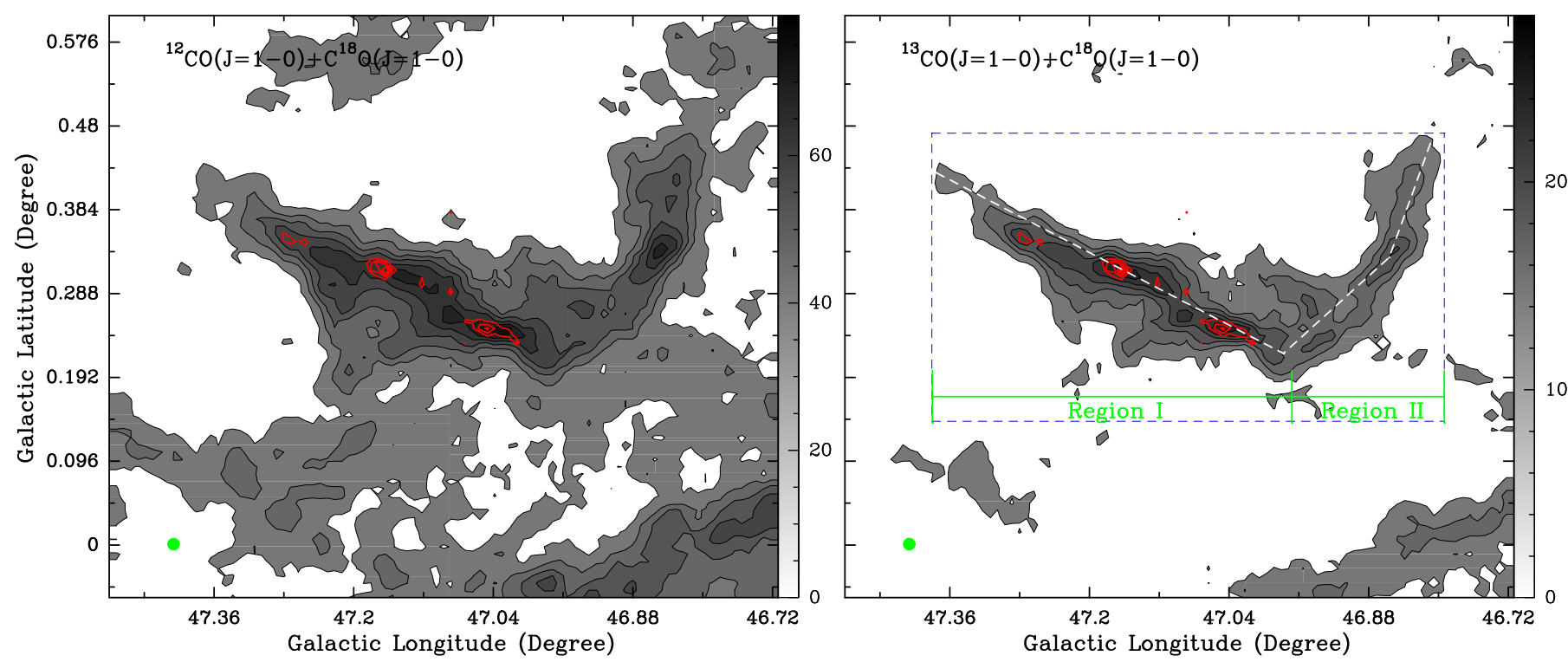

Fig. 2. Left panel: ${ }^{12} \mathrm{CO}(J=1-0)$ emission map (gray scale) overlaid with $\mathrm{C}^{18} \mathrm{O}(J=1-0)$ emission contours (red). The gray levels range from $11.0(5 \sigma)$ to 77.0 in steps of $11.0 \mathrm{~K} \mathrm{~km} \mathrm{~s}^{-1}$, while the red contour levels range from $3.0(5 \sigma)$ to 4.8 in steps of $0.6 \mathrm{~K} \mathrm{~km} \mathrm{~s}^{-1}$. Right panel: ${ }^{13} \mathrm{CO}(J=1-0)$ integrated emission map. The gray levels range from $3.0(5 \sigma)$ to 24.0 in steps of $4.2 \mathrm{~K} \mathrm{~km} \mathrm{~s}^{-1}$. In both maps, the emission has been integrated from 52 to $64 \mathrm{~km} \mathrm{~s}^{-1}$. The dashed box indicates our studied filament. The white dashed lines indicate the direction of position-velocity diagrams (see Fig. 9). The beam size is shown in the lower-left corner. The scale units in each panel of both axes are in degrees

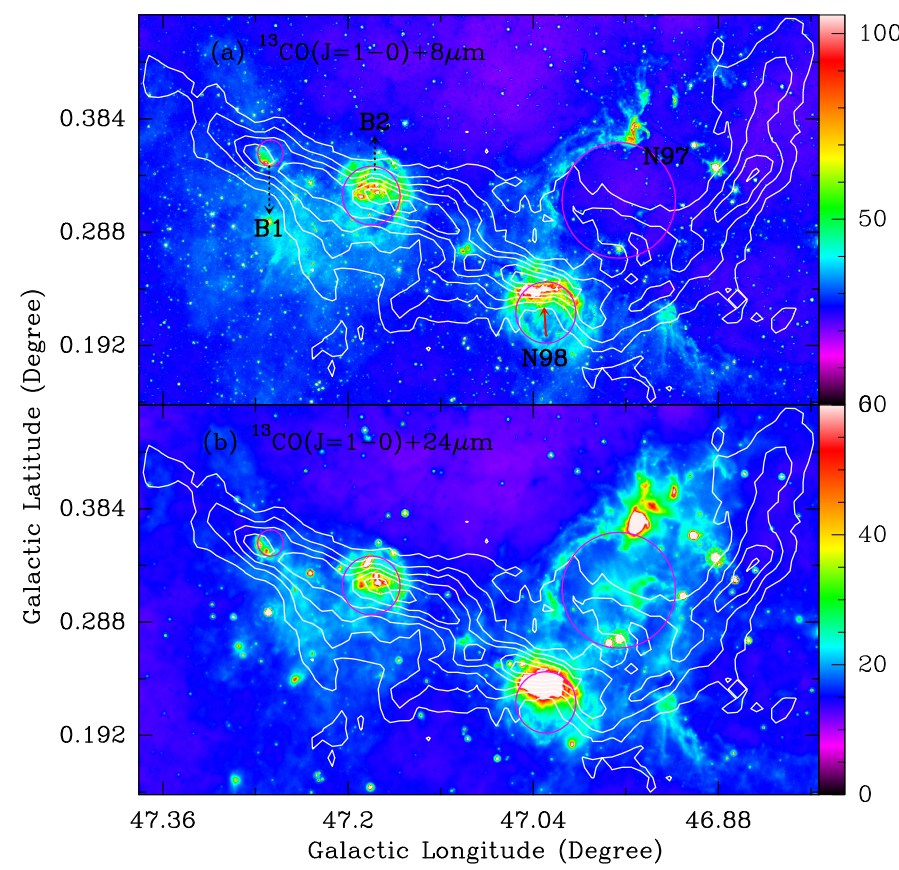

Fig. 3. Panel $a$ : ${ }^{13} \mathrm{CO} J=1-0$ emission map (white contours) superimposed on the Spitzer-IRAC $8 \mu \mathrm{m}$ emission map (color scale). The red arrow indicates steep integrated intensity gradients. Panel $b$ : ${ }^{13} \mathrm{CO} J=1-0$ emission map (white contours) superimposed on the Spitzer-MIPSGAL $24 \mu \mathrm{m}$ emission (color scale). The four pink circles represent infrared bubbles. B1 is bubble MWP1G047270+003500S, while B2 is MWP1G047180+003195. The right color-bar is in units of $\mathrm{mJy} \mathrm{sr}^{-1}$.

destroyed inside the ionized gas (Pomarès et al. 2009). Hence, the Spitzer-IRAC $8 \mu \mathrm{m}$ emission can be used to delineate an infrared bubble. Infrared bubbles are highlighted by the bright $8.0 \mu \mathrm{m}$ emission surrounding $\mathrm{O}$ and early- $\mathrm{B}$ stars, which show full or partial ring structures (Churchwell et al. 2006). Churchwell et al. (2006, 2007) compiled a list of 600 infrared bubbles, while Simpson et al. (2012) created a new catalog of 5106 infrared bubbles through visual inspection via the online citizen science website "The Milky Way Project (MWP)". From the above two catalogs, we identified four infrared bubbles N97, N98, MWP1G047270+003500S(B1), and MWP1G047180+003195(B2). We use pink circles to represent the bubbles in Fig. 3. N97 and N98 are close to the filamentary molecular cloud, while B1 and B2 are located within it. In order to clearly show the shape of a single bubble, we made the enlarged maps shown in Fig. 4. N97 shows an arc-like structure that is opened at the southwest, while N98 presents a cometary structure. From Fig. 3, we can see that the brightest part of N98 is located within the filamentary molecular cloud. CO molecular gas adjacent to N98 also shows an arc-like structure with a very intense emission towards N98, as shown by the red arrow in Fig. 3a. The H II region G047.028+0.232 is associated with N98 (Anderson et al. 2011). The RRL velocity of G047.028+0.232 is $56.9 \pm 0.1 \mathrm{~km} \mathrm{~s}^{-1}$, which is roughly consistent with the LSR velocity of the filamentary molecular cloud $\left(57.5 \mathrm{~km} \mathrm{~s}^{-1}\right)$. The $1.4 \mathrm{GHz}$ radio continuum emission is mainly from free-free emission, which can be used to trace the ionized gas of H II regions. Figure 4 also presents the radio $1.4 \mathrm{GHz}$ continuum emission maps overlaid on the Spitzer-IRAC $8 \mu \mathrm{m}$ emission. The infrared bubbles N97, N98, and B1 are associated with ionized gas, while there is no $1.4 \mathrm{GHz}$ radio continuum emission found for $\mathrm{B} 2$.

Figure 5 shows the ATLASGAL $870 \mu \mathrm{m}$ emission of the filament overlaid with the ${ }^{13} \mathrm{CO}(J=1-0)$ emission. The $870 \mu \mathrm{m}$ emission traces the distribution of cold dust. Obviously, the ${ }^{13} \mathrm{CO}(J=1-0)$ emission is more extended than that of the ATLASGAL $870 \mu \mathrm{m}$. Comparing with the $\mathrm{C}^{18} \mathrm{O}(J=1-0)$ emission in Fig. 2 (right panel), the spatial distribution of $870 \mu \mathrm{m}$ is similar to that of $\mathrm{C}^{18} \mathrm{O}(J=1-0)$, which is denser adjacent to N98. Next, BGPS is a $1.1 \mathrm{~mm}$ survey of dust emission in the Northern Galactic plane, which has a 33" beam (Ginsburg et al. 2013). These authors compiled a new catalog (v2.0), which includes 8594 sources. Most BGPS sources show signs of active 


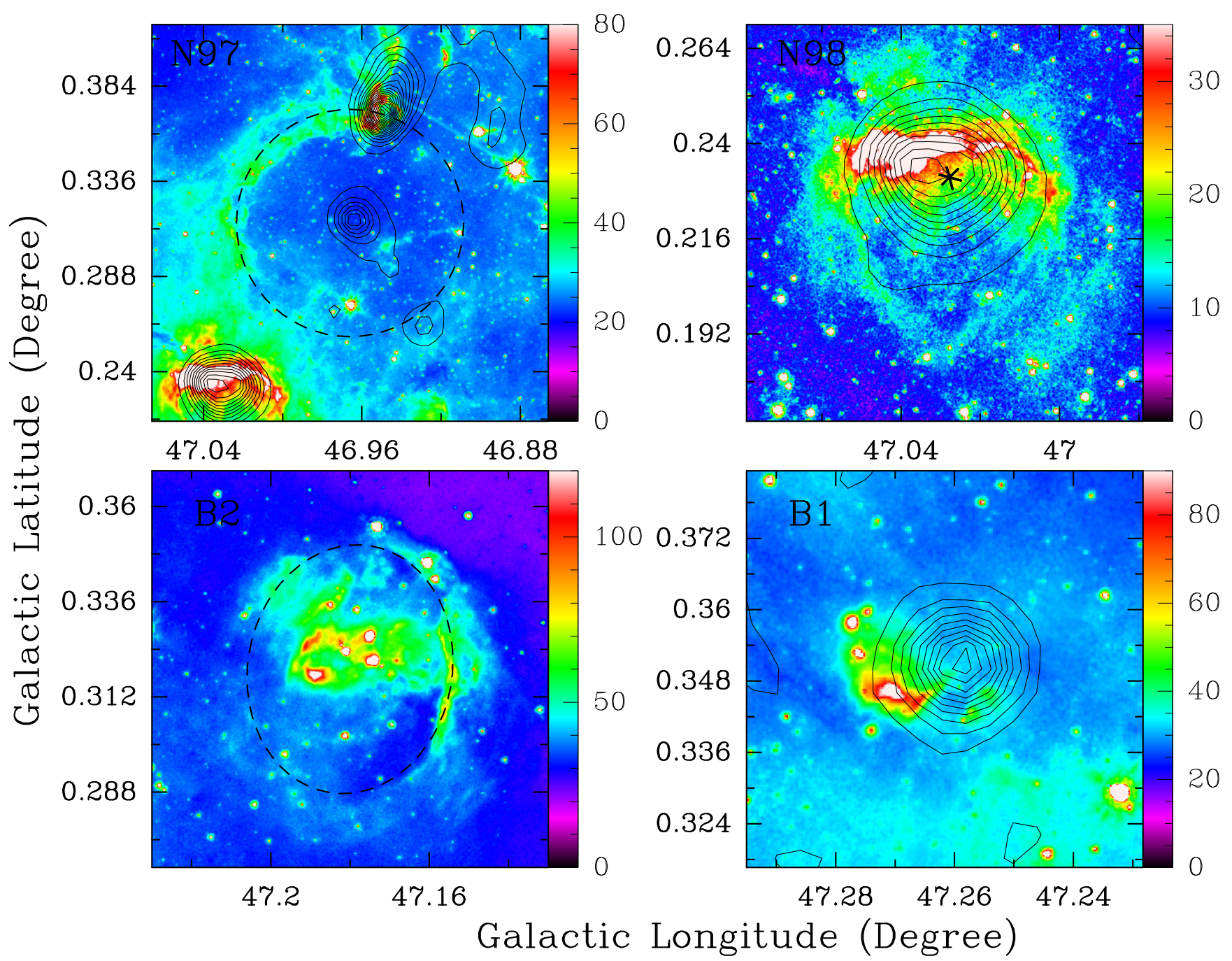

Fig. 4. Spitzer-IRAC $8 \mu \mathrm{m}$ emission maps (color scale) of Bubbles N97, N98, B2, and B1 overlaid with 1.4 GHz radio continuum emission contours (black). “*” indicates the position of H II region G047.028+0.232 (Anderson et al. 2011). The right color bar is in units of mJy sr ${ }^{-1}$.

star formation (Dunham et al. 2010; Xi et al. 2015). Using this catalog, we found ten BGPS sources in our observed region, as shown in Fig. 5. Except for one source, the remaining sources are all associated with ATLASGAL $870 \mu \mathrm{m}$ filamentary emission and ${ }^{13} \mathrm{CO}(J=1-0)$ emission. Table 2 lists the parameters of nine BGPS sources.

\subsection{Physical parameters}

\subsubsection{The column density and mass of G47.06+0.26}

To determine the column density and mass of the filamentary molecular cloud G47.06+0.26, we used the optically thin ${ }^{13} \mathrm{CO}(J=1-0)$ emission. Assuming local thermodynamical equilibrium (LTE), the column density was estimated via Garden et al. (1991)

$N\left({ }^{13} \mathrm{CO}\right)=4.71 \times 10^{13} \frac{T_{\mathrm{ex}}+0.88}{\exp \left(-5.29 / T_{\mathrm{ex}}\right)} \frac{\tau}{1-\exp (-\tau)} W \mathrm{~cm}^{-2}$,

where $T_{\mathrm{ex}}$ is the mean excitation temperature of the molecular gas and $\tau$ is the optical depth. $W$ is $\int T_{\mathrm{mb}} \mathrm{d} v$ in units of $\mathrm{K} \mathrm{km} \mathrm{s}^{-1}$, $\mathrm{d} v$ is the velocity range and $T_{\mathrm{mb}}$ is the corrected main-beam temperature of ${ }^{13} \mathrm{CO}(J=1-0)$.
Generally, the ${ }^{12} \mathrm{CO}$ emission is optical thick, so we used ${ }^{12} \mathrm{CO}$ to estimate $T_{\mathrm{ex}}$ via following the equation (Garden et al. 1991)

$T_{\mathrm{ex}}=\frac{5.53}{\ln \left[1+5.53 /\left(T_{\mathrm{mb}}+0.82\right)\right]}$,

where $T_{\mathrm{mb}}$ is the corrected main-beam brightness temperature of ${ }^{12} \mathrm{CO}$. Using all emission that is greater than $5 \sigma$, we made a map of the excitation temperature of ${ }^{12} \mathrm{CO}(J=1-0)$. In Fig. 6a, we find that the excitation temperature is $4.4-17.0 \mathrm{~K}$, while the mean excitation temperature of the filament is about $9.3 \mathrm{~K}$.

Moreover, we assumed that the excitation temperature of ${ }^{12} \mathrm{CO}$ and ${ }^{13} \mathrm{CO}$ have the same value in the filamentary cloud. The optical depth $(\tau)$ can be derived using the following equation (Garden et al. 1991)

$$
\tau\left({ }^{13} \mathrm{CO}\right)=-\ln \left[1-\frac{T_{\mathrm{mb}}}{5.29 /\left[\exp \left(5.29 / T_{\mathrm{ex}}\right)-1\right]-0.89}\right] .
$$

From the above equation, we made a map of the optical depths of ${ }^{13} \mathrm{CO}(J=1-0)$ (the $\mathrm{b}$ panel in Fig. 6). We derive that the optical depth is $0.1-1.0$, suggesting that the ${ }^{13} \mathrm{CO}$ emission is optically thin in the filament. 


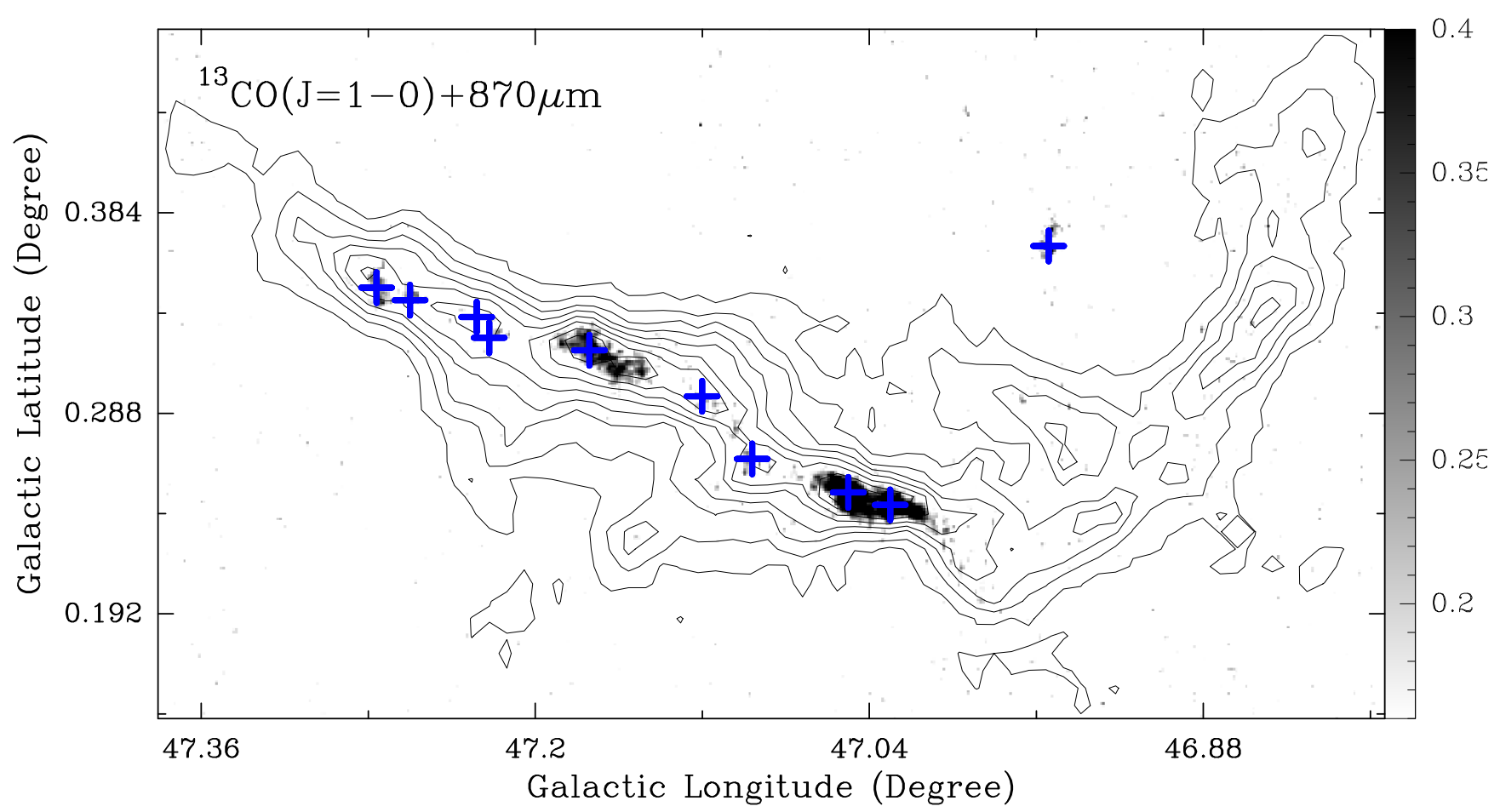

Fig. 5. ${ }^{13} \mathrm{CO}(J=1-0)$ integrated intensity map of the filament overlaid on the ATLASGAL $870 \mu \mathrm{m}$ image (gray scale). The blue pluses indicate the positions of $1.1 \mathrm{~mm}$ BGPS sources. The right color bar is in units of Jy beam ${ }^{-1}$.

Table 2. Physical parameters of the BGPS clumps.

\begin{tabular}{lcccccccc}
\hline \hline Name & $\begin{array}{c}l \\
(\mathrm{deg})\end{array}$ & $\begin{array}{c}b \\
(\mathrm{deg})\end{array}$ & $\begin{array}{c}L_{\text {maj }} \\
(\operatorname{arcsec})\end{array}$ & $\begin{array}{c}L_{\min } \\
(\operatorname{arcsec})\end{array}$ & $\begin{array}{c}\text { pos } \\
(\mathrm{deg})\end{array}$ & $\begin{array}{c}\text { Flux } \\
(\mathrm{Jy})\end{array}$ & $\begin{array}{c}M \\
\left(M_{\odot}\right)\end{array}$ & $\begin{array}{c}n\left(\mathrm{H}_{2}\right) \\
\left(10^{4} \mathrm{~cm}^{-3}\right)\end{array}$ \\
\hline G047.030+00.244 & 47.030 & 0.244 & 43.62 & 17.72 & 64 & 2.2 & 568.2 & 7.5 \\
G047.050+00.250 & 47.050 & 0.250 & 42.28 & 26.09 & 68 & 3.7 & 955.5 & 7.4 \\
G047.096+00.266 & 47.096 & 0.266 & 37.08 & 18.91 & 34 & 0.6 & 155.0 & 2.4 \\
G047.119+00.296 & 47.120 & 0.296 & 33.85 & 18.64 & 37 & 0.7 & 180.8 & 3.2 \\
G047.173+00.318 & 47.174 & 0.318 & 57.49 & 28.75 & 80 & 4.0 & 1033.0 & 4.4 \\
G047.222+00.324 & 47.222 & 0.324 & 32.87 & 19.84 & 117 & 0.8 & 206.6 & 3.5 \\
G047.228+00.334 & 47.228 & 0.334 & 19.12 & 17.82 & 170 & 0.5 & 129.1 & 5.8 \\
G047.259+00.342 & 47.260 & 0.342 & 38.77 & 18.62 & 16 & 0.9 & 232.4 & 3.4 \\
G047.276+00.348 & 47.276 & 0.348 & 28.80 & 22.41 & 43 & 1.1 & 284.1 & 4.9 \\
\hline
\end{tabular}

Notes. The first column is the name of the clumps. The second and third columns list the galactic longitude and latitude of the clumps, respectively. The fourth, fifth, and sixth columns list the angular sizes of major axis and minor axis, and the position angle of the clumps, respectively. The seventh, eighth, and ninth columns list the integrated flux density, mass, and number density of each clump, respectively.

Next we used the relation $N\left(\mathrm{H}_{2}\right) / N\left({ }^{13} \mathrm{CO}\right) \approx 7 \times 10^{5}$ (Castets et al. 1982) to estimate the $\mathrm{H}_{2}$ column density. We also made a map of the column density, as shown in Fig. 6c. The obtained column density is $(0.8-30.3) \times 10^{21} \mathrm{~cm}^{-2}$, while the mean column density is $6.2 \times 10^{21} \mathrm{~cm}^{-2}$. Generally, filaments show approximately long cylindrical structures (Jackson et al. 2010), thus the mass of the filament can be given by

$M_{\mathrm{H}_{2}}=\pi\left(\frac{d}{2}\right)^{2} l \mu_{\mathrm{g}} m\left(\mathrm{H}_{2}\right) n\left(\mathrm{H}_{2}\right)$,

where $\mu_{\mathrm{g}}=1.36$ is the mean atomic weight of the gas, $l$ is the length of the filament, $m\left(\mathrm{H}_{2}\right)$ is the mass of a hydrogen molecule, and $d$ is the mean width $(6.8 \mathrm{pc})$ of the filament. The mean number density of $\mathrm{H}_{2}$ is estimated to be $n\left(\mathrm{H}_{2}\right)=8.1 \times 10^{-20} N\left(\mathrm{H}_{2}\right) / d$ (Garden et al. 1991). Hence, we obtained that the mean number density of the filament is $147.2 \mathrm{~cm}^{-3}$. Using the mean number density, we derived a mass of $\sim 2.1 \times 10^{4} M_{\odot}$, which is approximatively equal to that obtained from Wang et al. (2015) for $\mathrm{G} 47.06+0.26$.

\subsubsection{The masses of BGPS clumps}

The dust continuum emission is expected to be optically thin at millimeter wavelengths in BGPS clumps (Bally et al. 2010). The masses of the BGPS clumps, as derived from their dust continuum emission, were determined using the relation

$M_{\text {clump }}=\frac{S_{v} D^{2}}{\kappa_{v} B_{v}\left(T_{\mathrm{d}}\right)}$

where $S_{v}$ is the flux density at the frequency $v$ and $D$ is the distance to the clumps. $\kappa_{v}$ is the dust opacity, which is adopted as $0.0114 \mathrm{~cm}^{2} \mathrm{~g}^{-1}$ (Enoch et al. 2006). Here the ratio of gas 


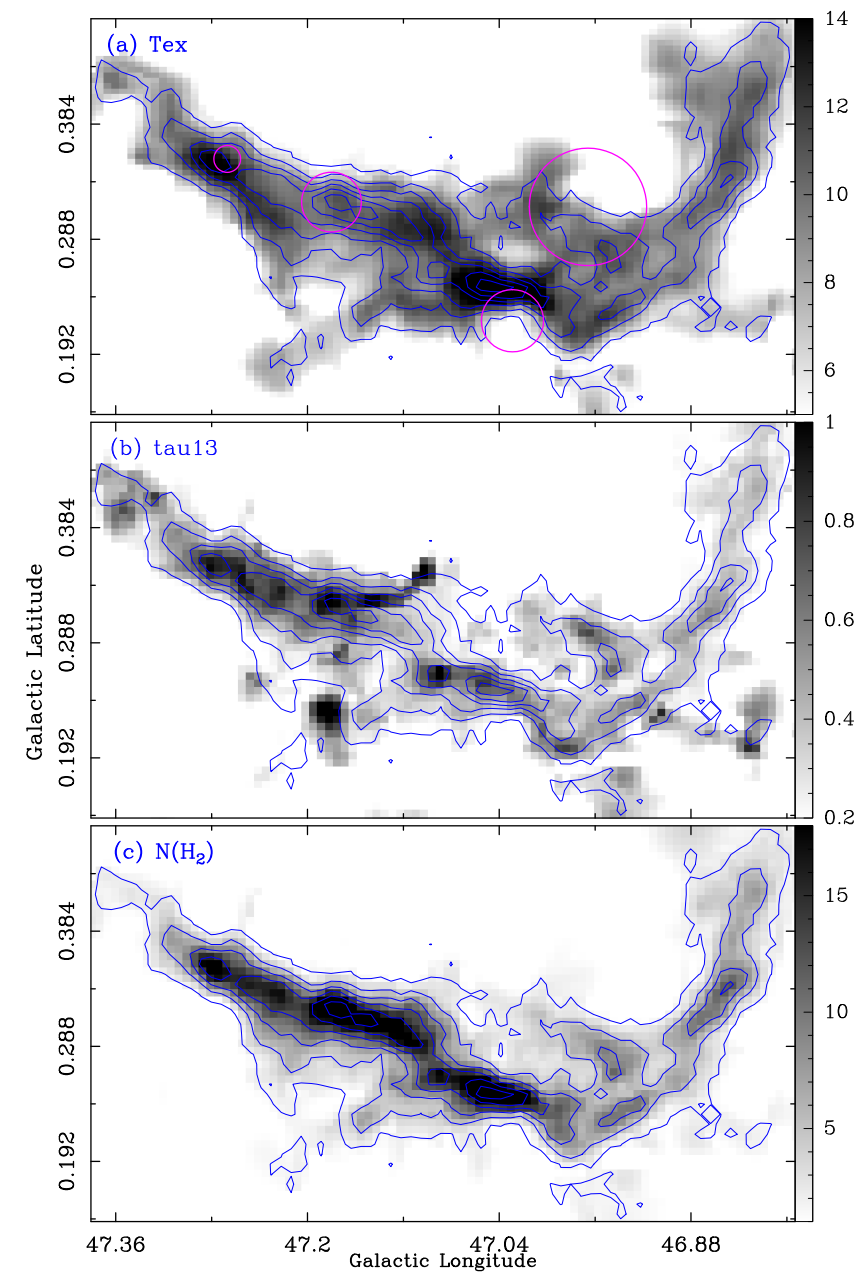

Fig. 6. Panel $a$ : map of the excitation temperature with a linear scale in units of $\mathrm{K}$ from the ${ }^{12} \mathrm{CO}(J=1-0)$ emission in the $52-64 \mathrm{~km} \mathrm{~s}^{-1}$ interval. Panel $b$ : map of the optical depths of ${ }^{13} \mathrm{CO}(J=1-0)$. Panel $c$ : map of the column density in units of $10^{21} \mathrm{~cm}^{-2}$. The blue contours represent the ${ }^{13} \mathrm{CO}(J=1-0)$ emission map.

to dust was taken as 100 , which is included in $\kappa_{v} . B_{v}\left(T_{\mathrm{d}}\right)$ is the Planck function for the dust temperature $T_{\mathrm{d}}$ and frequency $v$. According to Rosolowsky et al. (2010), we also used a dust temperature of $20 \mathrm{~K}$ for each BGPS clump. Assuming that the clumps have roughly spherical shapes, we computed the $\mathrm{H}_{2}$ volume density via $n\left(\mathrm{H}_{2}\right)=3 M_{\text {clump }} /\left(4 \pi r^{3} \mu_{\mathrm{g}} m\left(\mathrm{H}_{2}\right)\right)$, where $r$ is the effective radius of each clump, which can be determined by $r=$ $\sqrt{L_{\text {maj }} \cdot L_{\text {min }}} / 2$. Here, $L_{\text {maj }}$ and $L_{\text {min }}$ are the de-convolved major and minor axes of each clump, which are listed in Table 2. The obtained masses and volume densities of these BGPS clumps are listed in Table 2. From Table 2, we found that these clumps have masses ranging from 129 to $1033 M_{\odot}$ with a total mass of clumps of $3.8 \times 10^{3} M_{\odot}$.

\subsubsection{The ionizing luminosity and ages of the $\mathrm{H} \|$ regions}

Four infrared bubbles are found to be close to the filament. Infrared bubbles N98 and B1 are filled with ionized gas, which is traced by the $1.4 \mathrm{GHz}$ radio continuum emission, suggesting there are two $\mathrm{H}$ II regions located within the centers of these two bubbles. Only a small region of ionized gas is detected at the center of N97. There is no detected ionized gas in B2. Thus, we only calculated the dynamical ages of $\mathrm{H}$ II regions associated with $\mathrm{N} 98$ and $\mathrm{B} 1$. Using the $1.4 \mathrm{GHz}$ radio continuum emission, the ionizing luminosity $N_{\text {Ly }}$ was computed via (Condon 1992)

$N_{\text {Ly }}=7.54 \times 10^{46}\left(\frac{v}{\mathrm{GHz}}\right)^{0.1}\left(\frac{T_{4}}{\mathrm{~K}}\right)^{-0.45}\left(\frac{S_{v}}{\mathrm{Jy}}\right)\left(\frac{D}{\mathrm{kpc}}\right)^{2} \mathrm{~s}^{-1}$,

where $v$ is the frequency of the observation, $T_{4}$ is the effective electron temperature in units of $10^{4} \mathrm{~K}, S_{v}$ is the observed specific flux density, and $D$ is the distance to the H II region. Here we adopted an effective electron temperature of $10^{4} \mathrm{~K}$. Because both N98 and B1 are interacting with the filament (see Sect. 4.1), we took the distance $(4.44 \mathrm{kpc})$ of the filament as those of the two H II regions. From Fig. 4, we measured a flux density of $630 \mathrm{mJy}$ and $24 \mathrm{mJy}$ at $1.4 \mathrm{GHz}$ for the two $\mathrm{HII}$ regions, respectively. Finally, we derived $N_{\mathrm{Ly}} \simeq 9.7 \times 10^{47} \mathrm{ph} \mathrm{s}^{-1}$ and $3.7 \times 10^{46} \mathrm{ph} \mathrm{s}^{-1}$ for the two H II regions.

Assuming an HII region expanding in a homogeneous medium, the dynamical age was estimated by Dyson \& Williams (1980)

$t_{\mathrm{HII}}=\frac{4 R_{\mathrm{s}}}{7 c_{\mathrm{s}}}\left[\left(\frac{R_{\mathrm{HII}}}{R_{\mathrm{s}}}\right)^{7 / 4}-1\right]$

where $c_{\mathrm{s}}$ is the isothermal sound speed of ionized gas, assumed to be $10 \mathrm{~km} \mathrm{~s}^{-1}, R_{\mathrm{HII}}$ is the radius of the $\mathrm{HII}$ region, and $R_{\mathrm{S}}$ is the radius of the Strömgren sphere given by $R_{\mathrm{S}}=\left(3 N_{\mathrm{Ly}} / 4 \pi n_{i}^{2} \alpha_{B}\right)^{1 / 3}$, where $N_{\text {Ly }}$ is the ionizing luminosity, $n_{\mathrm{i}}$ is the initial $\mathrm{H}$ number density of the gas, and $\alpha_{B}=2.6 \times 10^{-13} \mathrm{~cm}^{3} \mathrm{~s}^{-1}$, which is the hydrogen recombination coefficient to all levels above the ground level. We used the volume-averaged $\mathrm{H}_{2}$ density $\left(147.2 \mathrm{~cm}^{-3}\right)$ of the filament to determine $n_{\mathrm{i}}$ since the two H II regions are located in or close to the filament. Adopting a radius of $\sim 1.6 \mathrm{pc}$ and $\sim 0.6 \mathrm{pc}$ for the two H II regions obtained from Fig. 4, we found that the dynamical ages are $6.3 \times 10^{5} \mathrm{yr}$ and $3.9 \times 10^{5} \mathrm{yr}$ for the two H II regions associated with N98 and B1, respectively.

Infrared bubbles were found to be associated with the filament. The bubbles will inject kinetic energy into the filament. According to Lasker (1967), we estimate the kinetic energy of N98 and B1 by

$E_{\mathrm{k}}=\frac{4}{3} \pi n_{i} m_{\mathrm{H}} c_{\mathrm{s}}^{2} R_{\mathrm{s}}^{3 / 2}\left[\left(\frac{7}{4} c_{\mathrm{s}} R_{\mathrm{s}}^{3 / 4} t+R_{\mathrm{s}}^{7 / 4}\right)^{6 / 7}-R_{\mathrm{s}}^{3 / 2}\right]$,

where $m_{\mathrm{H}}$ is the mass of a hydrogen atom, and $t$ represents the dynamical age of the H II region. As both N98 and B1 surround an $\mathrm{H}$ II region, we can estimate the ionization energy and thermal energy in the H II regions (Freyer et al. 2003) as:

$E_{\mathrm{i}}=\frac{4}{3} \pi n_{i}\left(\frac{7}{4} c_{\mathrm{s}} R_{\mathrm{s}}^{5 / 2} t+R_{\mathrm{s}}^{7 / 2}\right)^{6 / 7} \chi_{0}$,
$E_{\mathrm{t}}=\frac{4}{3} \pi n_{i}\left(\frac{7}{4} c_{\mathrm{s}} R_{\mathrm{s}}^{5 / 2} t+R_{\mathrm{s}}^{7 / 2}\right)^{6 / 7} k T_{\mathrm{II}}$,

where $\chi_{0}$ is the ionization potential $(13.6 \mathrm{eV})$ of hydrogen in the ground state, and $T_{\mathrm{II}}$ is the effective electron temperature $\left(10^{3} \mathrm{~K}\right)$ in the H II regions. The obtained results are listed in Table 3. From Table 3, we can see that the ionization energy is one order of magnitude higher than kinetic energy and thermal energy. 


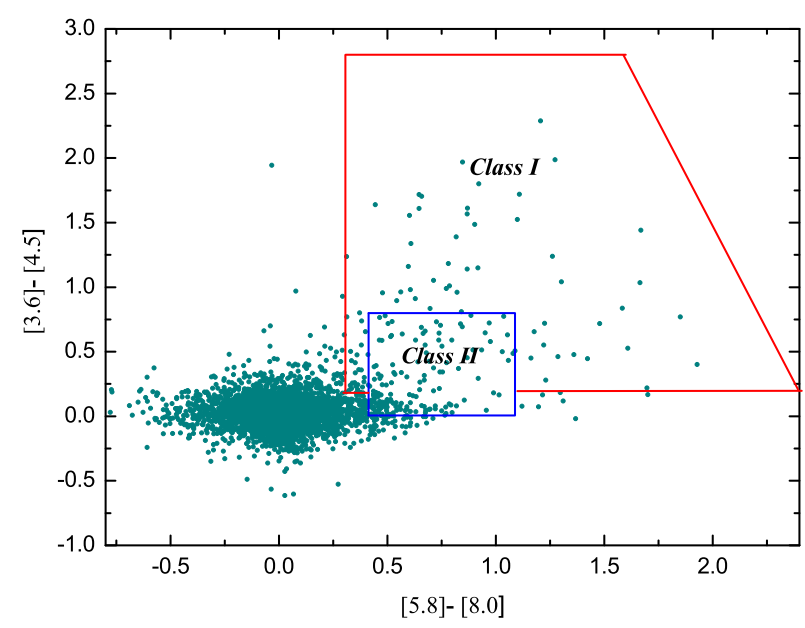

Fig. 7. GLIMPSE color-color diagram [5.8]-[8.0] vs. [3.6]-[4.5] for all the selected sources in filament G47.06+0.26. Class I and II regions are indicated according to criteria given by Allen et al. (2004). Class I sources are protostars with circumstellar envelopes and Class II are disk-dominated objects.

Table 3. Energy components of H II regions.

\begin{tabular}{lcccc}
\hline \hline Name & $\begin{array}{c}E_{k} \\
\left(\times 10^{47} \mathrm{erg}\right)\end{array}$ & $\begin{array}{c}E_{i} \\
\left(\times 10^{47} \mathrm{erg}\right)\end{array}$ & $\begin{array}{c}E_{t} \\
\left(\times 10^{47} \mathrm{erg}\right)\end{array}$ & $\begin{array}{c}t_{\mathrm{HII}} \\
\left(\times 10^{5} \mathrm{yr}\right)\end{array}$ \\
\hline N98 & $2.2 \pm 0.7$ & $31.6 \pm 0.9$ & $2.0 \pm 0.6$ & 6.3 \\
B1 & $0.1 \pm 0.03$ & $2.0 \pm 0.5$ & $0.1 \pm 0.04$ & 3.9 \\
\hline
\end{tabular}

Notes. The first, second, and third columns are the kinetic energy, ionization energy and thermal energy, respectively. The fourth column is the dynamical age.

\subsection{Distribution of young stellar objects}

To investigate the star formation activity in the filament, we use the GLIMPSE I Spring'07 catalog (highly reliable) to select young stellar objects (YSOs). Using a relation of $A_{v}=5.34 \times 10^{-22} N\left(\mathrm{H}_{2}\right)$ (Deharveng et al. 2009) and adopting a mean column density of $6.2 \times 10^{21} \mathrm{~cm}^{-2}$, we obtain an extinction of $3.3 \mathrm{mag}$ in filament G47.06+0.26. The IRAC [5.8]-[8.0] vs. [3.6]-[4.5] color-color (CC) diagram is a useful tool for identifying YSOs with infrared excess (Allen et al. 2004). The effects of extinction are relatively small at these wavelengths. Using the criteria of Allen et al. (2004), we selected near-infrared sources with 3.6, 4.5, 5.8, and $8.0 \mu \mathrm{m}$ detections in our observed region from the catalog. Figure 7 shows the [5.8]-[8.0] vs. [3.6]-[4.5] color-color (CC) diagram, which can be used to classify nearinfrared sources into three regions: class I YSOs are protostars with circumstellar envelopes, class II YSOs are disk-dominated objects, and other sources. Class I YSOs have a timescale of the order of $\sim 10^{5} \mathrm{yr}$, while the age of class II YSOs is $\sim 10^{6} \mathrm{yr}$ (André \& Montmerle 1994). Based on this criteria, we selected 75 Class I YSOs and 179 class II YSOs.

The spatial distribution of these YSOs is shown in Fig. 8. To display the positional relation of YSOs with the filament, we also overlaid the selected class I and class II YSOs on the ${ }^{13} \mathrm{CO}(J=1-0)$ emission (gray scale). We note that class II YSOs (blue dots) appear to be dispersedly distributed across the whole selected region. Class I sources (red dots) are found to be evenly along regions of high column density, which are mostly concentrated in the filamentary molecular cloud. In addition, region I of the filament has a higher concentration of class I YSOs than in the region II. To further confirm above the results,

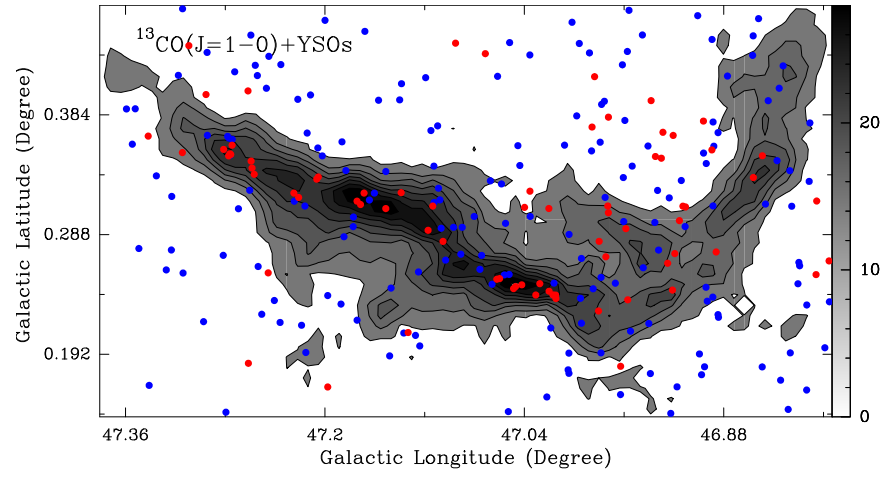

Fig. 8. Positions of class I and II YSOs relative to the ${ }^{13} \mathrm{CO}(J=1-0)$ emission (gray scale). Class I YSOs are labeled as red dots, and class II YSOs as blue dots.

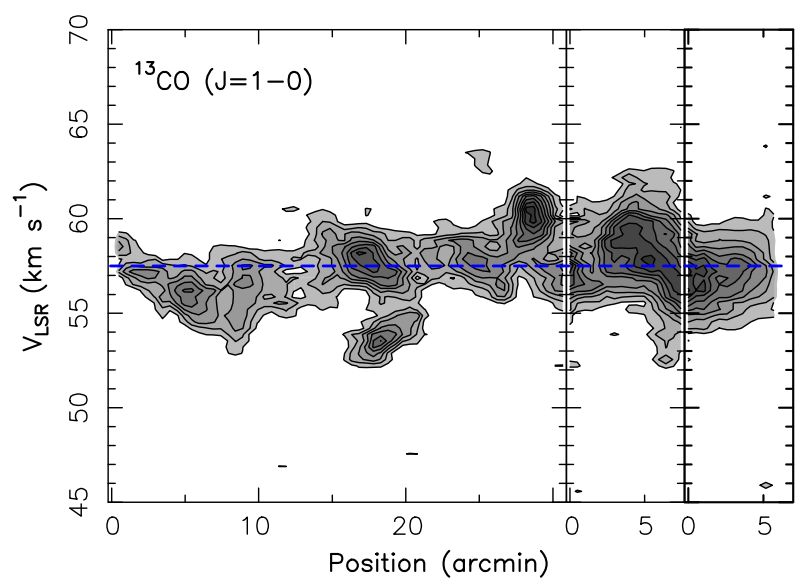

Fig. 9. Position-velocity diagrams of the ${ }^{13} \mathrm{CO}(J=1-0)$ emission along filament G47.06+0.26 (see the long dashed lines in the right panel of Fig. 2). The blue dashed line marks an LSR velocity of $57.5 \mathrm{~km} \mathrm{~s}^{-1}$.

we calculated the surface densities of the class I and class II YSOs inside and outside of the filament. For the filament, the surface density of class I YSOs is $0.12 \mathrm{pc}^{-2}$. The region outside the filament has a surface density of $0.04 \mathrm{pc}^{-2}$, which represents the surface density of the total foreground and background class I YSOs. Getting rid of the total foreground and background objects, then we obtained a surface density of $0.08 \mathrm{pc}^{-2}$ for the filament. Because we only selected the near-infrared sources with the $3.6,4.5,5.8$, and $8.0 \mu \mathrm{m}$ emission, a surface density of $0.08 \mathrm{pc}^{-2}$ may be a lower limit. Moreover, the filament has a surface density of $0.17 \mathrm{pc}^{-2}$ for class II YSOs, which is approximately equal to the value $\left(0.15 \mathrm{pc}^{-2}\right)$ outside of the filament, indicating that the class II YSOs are evenly distributed along the whole region.

\section{Discussions}

\subsection{Dynamic structure of the filament}

From the ${ }^{12} \mathrm{CO}(J=1-0)$ and ${ }^{13} \mathrm{CO}(J=1-0)$ emission, $\mathrm{G} 47.06+0.26$ shows a filamentary structure. The region traced by the ${ }^{12} \mathrm{CO}(J=1-0)$ emission is more diffuse than that of ${ }^{13} \mathrm{CO}(J=1-0)$ in $\mathrm{G} 47.06+0.26$. The $\mathrm{C}^{18} \mathrm{O}(J=1-0)$ emission is weaker, which is consistent with the ATLASGAL $870 \mu \mathrm{m}$ emission. The filamentary structure can be divided into two regions (region I and region II), as shown in Fig. 2. We do not detect $\mathrm{C}^{18} \mathrm{O}(J=1-0)$ and $870 \mu \mathrm{m}$ emission in region II. The $\mathrm{C}^{18} \mathrm{O}(J=1-0)$ and $870 \mu \mathrm{m}$ emission likely traces the densest regions of the filament. Figure 9 shows a position-velocity 


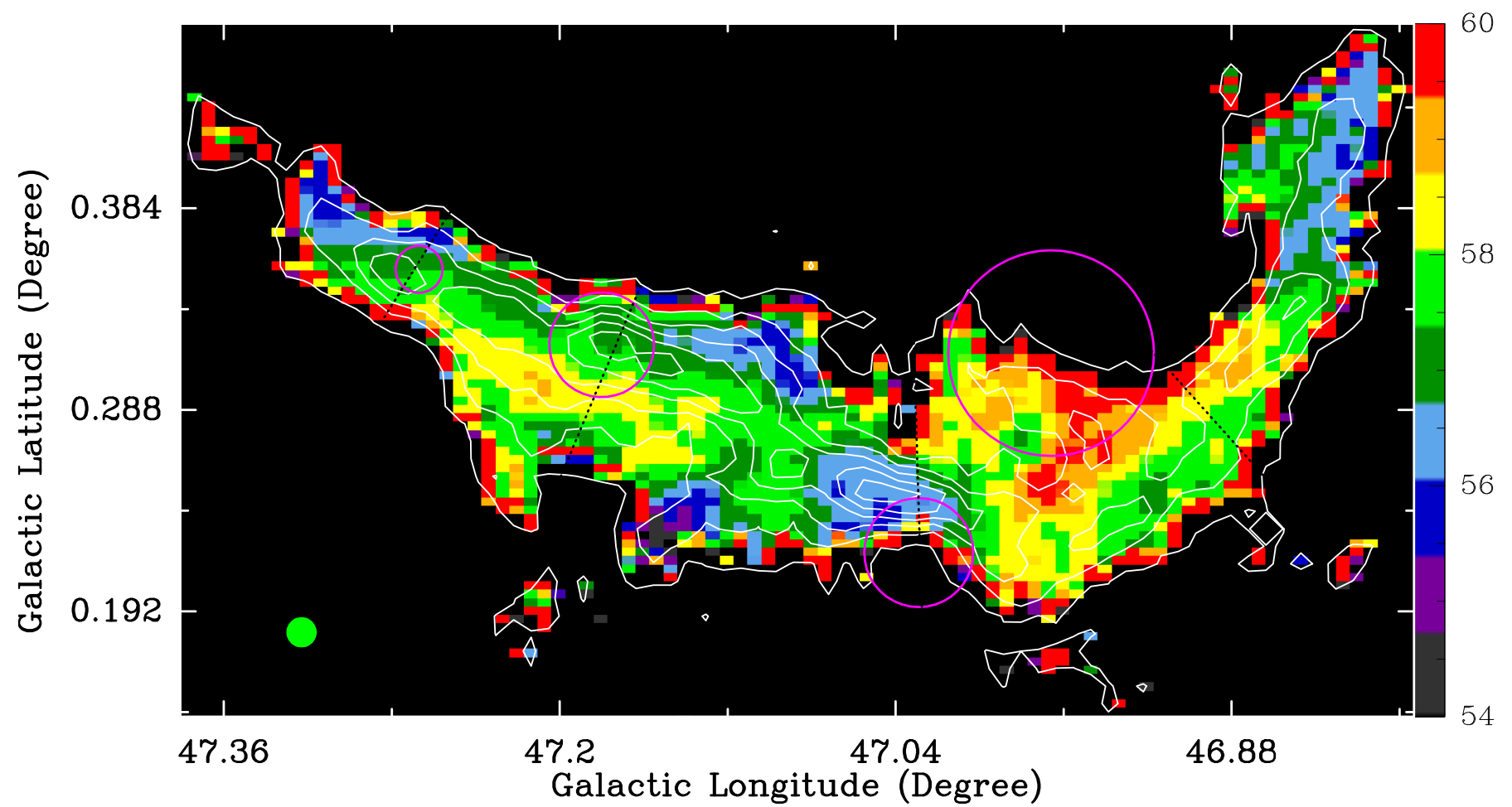

Fig. 10. Velocity-field (moment 1) map of ${ }^{13} \mathrm{CO}(J=1-0)$ overlaid with its integrated intensity contours (white). The four pink circles represent the identified infrared bubbles. The beam size is shown in the lower-left corner. The right color bar is in units of $\mathrm{km} \mathrm{s}^{-1}$. The four black dashed lines show the velocity gradients perpendicular to filament G47.06+0.26.

(PV) diagram constructed from the ${ }^{13} \mathrm{CO}(J=1-0)$ emission along the long filament G47.06+0.26. From this figure, we found that the filament not only shows a single coherent object, but also contains several ${ }^{13} \mathrm{CO}(J=1-0)$ clumps. Generally, filamentary molecular clouds harbor over-densities along their length (Contreras et al. 2016). Recently several authors have identified the signatures of gas flows along filaments (e.g., Peretto et al. 2013; Kirk et al. 2013; Tackenberg et al. 2014; Zhang et al. 2015). Because gas can be funneled along filaments and feed star-forming regions, filamentary flows may play a key role in star formation. In numerical simulations, and in comparison with cores in other regions, cores that are embedded within filaments have been shown to form more massive stars by accretion along filamentary flows (Smith et al. 2011). Figure 10 presents a velocity-field (Moment 1$)$ map of ${ }^{13} \mathrm{CO}(J=$ $1-0)$ of $\mathrm{G} 47.06+0.26$ overlaid with its integrated intensity contours. From the velocity-field map, we can discern a velocity gradient perpendicular to filament G47.06+0.26, but not along the filament. Fernández-López et al. (2014) suggested that such velocity-gradients, perpendicular to filaments may be due to motions associated with the formation and growth of the filaments within magnetized and turbulent sheet-like structures. As suggested by Smith et al. (2016), the formation of filaments is a natural consequence of turbulent cascades in the complex multiphase interstellar medium. Here we can estimate the turbulent energy of the filament G47.06+0.26, which is given by

$E_{\text {turb }}=\frac{1}{2} M \sigma_{3 \mathrm{~d}}^{2}$,

where $\sigma_{3 \mathrm{~d}} \approx \sqrt{3} \sigma_{v}$, which is the three-dimensional turbulent velocity dispersion, $\sigma_{v}$ is $\Delta V_{\mathrm{FWHM}} /(2 \sqrt{2 \ln 2})$, and $\Delta V_{\mathrm{FWHM}}$ is the mean full width at half-maximum (FWHM) of the ${ }^{13} \mathrm{CO}(J=$ $1-0)$ emission. In the filament, we found the mean FWHM to be $3.8 \mathrm{~km} \mathrm{~s}^{-1}$. The mean thermal broadening can be given by $\Delta V_{\text {therm }}=2.355 \times \sqrt{k T_{\text {ex }} /\left(\mu_{\mathrm{g}} m\left(\mathrm{H}_{2}\right)\right)}$. Adopting an excitation temperature $\left(T_{\text {ex }}\right)$ of $4.4-17.0 \mathrm{~K}$, we derived $\Delta V_{\text {therm }} \approx 0.3-$ $0.5 \mathrm{~km} \mathrm{~s}^{-1}$, which is much less than the mean FWHM of $3.8 \mathrm{~km} \mathrm{~s}^{-1}$ measured for ${ }^{13} \mathrm{CO}(J=1-0)$. Hence, we did not consider the velocity dispersion to be caused by thermal broadening. Using a mass of $\sim 2.1 \times 10^{4} M_{\odot}$ for the filament, we determined the turbulent energy of the filament to be about $1.6 \times 10^{48} \mathrm{erg}$.

Massive stars are a possible mechanism for driving turbulence through feedback from outflows, UV radiation fields, stellar winds, and supernova explosions in molecular clouds. Molecular outflows and supernova explosions have not been detected in G47.06+0.26. Instead, we found four infrared bubbles (N97, N98, B1, and B2) in this region. N98 shows a comet-like structure at $8 \mu \mathrm{m}$. The H II region G047.028+0.232 is associated with N98 (Anderson et al. 2011). The RRL velocity of G047.028+0.232 is roughly consistent with that of $\mathrm{G} 47.06+0.26$. Moreover, $\mathrm{CO}$ molecular gas of $\mathrm{G} 47.06+0.26$ shows an arc-like structure with a very intense emission towards N98. Hence, we conclude that N98 is interacting with filament G47.06+0.26. From the velocity-field map (Fig. 10), we found that the adjacent proximity of G47.06+0.26 to N97 and N98 is the likely source of the velocity gradient. Moreover, the direction of the velocity gradients are towards N97 and N98. Hence, the identified infrared bubbles are likely to be part of the turbulent sources in filament G47.06+0.26. We estimated the kinetic energy, ionization energy, and thermal energy of the H II region associated with N98. Both the obtained kinetic energy and thermal energy are far smaller than the turbulent energy $(1.6 \times$ $10^{48} \mathrm{erg}$ ) of filament G47.06+0.26. Compared to the kinetic energy and thermal energy, the ionization energy $\left(3.2 \times 10^{48} \mathrm{erg}\right)$ may inject more energy to G47.06+0.26, and help maintain the turbulence. 


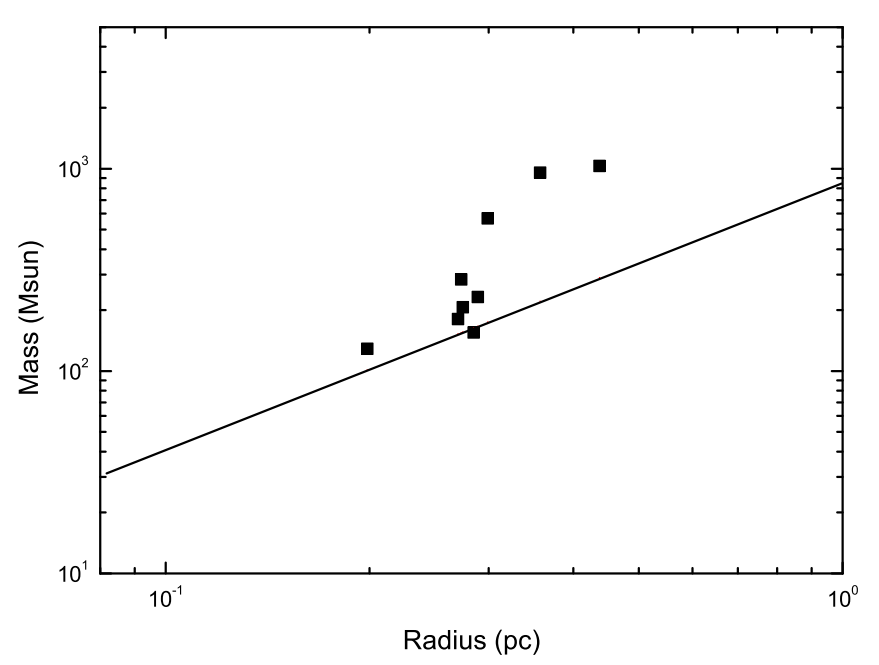

Fig. 11. Mass vs. radius for the BGPS sources embedded in the filament. The black line delineates the threshold (Kauffmann \& Pillai 2010), which is used to separate the regimes under which massive stars can form (above the line) or not (below the line).

\subsection{Fragmentation and star formation}

Using the mass and length $(\sim 58.1 \mathrm{pc})$ of the filament, we derived a linear mass density $M / l$ of $\sim 361.5 M_{\odot} \mathrm{pc}^{-1}$, which is an upper limit due to inclination and projection effects. If turbulence is dominant, a critical mass to length ratio can be estimated as $(M / l)_{\text {crit }}=84(\Delta V)^{2} M_{\odot} \mathrm{pc}^{-1}$ (Jackson et al. 2010). Then, we obtain $(M / l)_{\text {crit }} \approx 1.2 \times 10^{3} M_{\odot} \mathrm{pc}^{-1}$, which is larger than $M / l$. Hence, we conclude that external pressure (due to the neighboring bubbles and $\mathrm{H}$ II regions) may help prevent the filament from dispersing under the effects of turbulence.

From the BGPS catalog, we found ten BGPS sources in our observed region. In previous studies of BGPS sources, most show signs of active star formation (Dunham et al. 2010; $\mathrm{Xi}$ et al. 2015). We cannot say if star formation is ongoing from our data. To determine whether the BGPS sources have sufficient mass to form massive stars, we must consider their sizes and mass. According to Kauffmann \& Pillai (2010), if the clump mass is $M(r) \geq 870 M_{\odot}(r / p c)^{1.33}$, then they can potentially form massive stars. Figure 11 presents a mass vs. radius plot of the BGPS sources embedded in the filament. We find that all the BGPS sources lie at or above the threshold, indicating that these clumps are dense and massive enough to potentially form massive stars. Nine out of the ten sources are associated with ATLASGAL $870 \mu \mathrm{m}$ emission of G47.06+0.26. Although G47.06+0.26 is not gravitationally bound from the ${ }^{13} \mathrm{CO}(J=1-0)$ observation, the space distribution of the BGPS sources suggests that fragmentation may happen in the densest part of the filament. The total mass of filament G47.06+0.26 is $\sim 2.1 \times 10^{4} M_{\odot}$, as derived from the ${ }^{13} \mathrm{CO}(J=1-0)$ observation. These BGPS sources have a total mass of $3.8 \times 10^{3} M_{\odot}$. Hence, we can calculate a massive clump formation efficiency (CFE). The CFE is used to measure what fraction of molecular gas has been converted into dense clumps, which is given by $M_{\text {clump }} / M_{\text {cloud }}$ (Eden et al. 2012). The obtained CFE in the filament $\mathrm{G} 47.06+0.26$ is $\sim 18 \%$. In W3 giant molecular cloud (GMC), it is $26-37 \%$ in the compressed region by $\mathrm{H}$ II regions and only 5-13\% in the diffuse cloud (Moore et al. 2007). Moreover, Eden et al. (2013) used BGPS sources and Galactic Ring Survey data $\left({ }^{13} \mathrm{CO}\right)$ to check CFE changes with Galactic environment. They found that the total CFE values in the interarm and spiral-arm regions are about $4.9 \%$ and $5.5 \%$, but greater than $40 \%$ in the small-scale regions associated with $\mathrm{H}$ II regions. The increases in the CFE are related to local triggering due to feedback (Eden et al. 2013). Using the same data as (Eden et al. 2012, 2013), Battisti \& Heyer (2014) derived that the mean CFE is about $11 \%$ in the GMC of the Milky Way. Our obtained CFE value $(\sim 18 \%)$ is greater than those in the GMC of the Milky Way, and in the interarm and spiral-arm regions. For the L1641 filamentary molecular cloud in Orion, the CFE value is only $12 \%$ (Polychroni et al. 2013). The filament G47.06+0.26 is found to be associated with four infrared bubbles (N97, N98, B1, and B2). We suggest that the feedback from H II regions may be responsible for the higher CFE in the filament G47.06+0.26.

In addition, the bubbles can be produced by stellar wind and overpressure via ionization and heating by stellar UV radiation from $\mathrm{O}$ - and early-B stars. The presence of the infrared bubbles, suggests that several massive stars have formed in or near to the filament. The ionizing stars in these bubbles may be the first generation of stars that form in such a filament. The formation of the bubbles can impact their environments, and in particular they can trigger the next generation of star formation (Elmegreen 1998). We concluded that N98 is interacting with filament G47.06+0.26. From the GLIMPSE I catalog, we selected class I YSOs with ages of $\sim 10^{5} \mathrm{yr}$ on the basis of their infrared color indices. It is interesting to note that class I YSOs (red dots) are found to be distributed along regions of high column density, which are mostly concentrated in the filamentary molecular cloud. Moreover, we also find that the region adjacent to N98 contains more class I YSOs. The estimated dynamical ages are $6.3 \times 10^{5} \mathrm{yr}$ and $3.9 \times 10^{5} \mathrm{yr}$ for the two $\mathrm{H}$ II regions associated with N98 and B1, which are slightly greater than that of class I YSOs. A possible picture would be that the expansion of the $\mathrm{H}$ II regions then compressed the filament, and triggered the formation of a new generation of stars, including what is now seen as class I YSOs in filament G47.06+0.26.

\section{Conclusions}

We present molecular ${ }^{12} \mathrm{CO}(J=1-0),{ }^{13} \mathrm{CO}(J=1-0)$ and $\mathrm{C}^{18} \mathrm{O}(J=1-0)$, infrared, and radio continuum observations of filament G47.06+0.26. Molecular observations were obtained with the PMO $13.7 \mathrm{~m}$ radio telescope to investigate the detailed distribution of molecular material in G47.06+0.26. Our main results are summarized as follows:

1. From the ${ }^{12} \mathrm{CO}(J=1-0)$ and ${ }^{13} \mathrm{CO}(J=1-0)$ emission, $\mathrm{G} 47.06+0.26$ shows a filamentary structure. The ${ }^{12} \mathrm{CO}(J=$ $1-0)$ emission is more diffuse than that of the ${ }^{13} \mathrm{CO}(J=$ $1-0)$ in $\mathrm{G} 47.06+0.26$. The $\mathrm{C}^{18} \mathrm{O}(J=1-0)$ emission is weaker, which is consistent with the ATLASGAL $870 \mu \mathrm{m}$ emission. The filament extends by about $45.0^{\prime}$ along the eastwest direction, whose mean width is about $6.8 \mathrm{pc}$, as traced by the ${ }^{13} \mathrm{CO}(J=1-0)$ emission. The mass and mean number density of the filament are $\sim 2.1 \times 10^{4} M_{\odot}$ and $147.2 \mathrm{~cm}^{-3}$, respectively.

2. In the filament, we obtained a linear mass density of $\sim 361.5 M_{\odot} \mathrm{pc}^{-1}$, which is smaller than its critical mass to length ratio, suggesting that external pressure (due to the neighboring bubbles and H II regions) may help prevent the filament from dispersing under the effects of turbulence. The velocity-field (Moment 1) map shows a clear velocity gradient perpendicular to filament $\mathrm{G} 47.06+0.26$, but not along the filament. Such velocity-gradients perpendicular to filaments may stem from the filament-formation process within magnetized and turbulent structures. 
3. We determined that the turbulent energy of the filament is about $6.5 \times 10^{48} \mathrm{erg}$. Four infrared bubbles are found to be located in, or adjacent to, G47.06+0.26 along the line of sight. Particularly, the infrared bubble N98 shows a cometary structure and is expanding into G47.06+0.26. N98 is associated with $\mathrm{H}$ II region $\mathrm{G} 047.028+0.232$, with an age of $6.3 \times 10^{5} \mathrm{yr}$. We estimated the kinetic energy, ionization energy, and thermal energy of H II G047.028+0.232. Both the obtained kinetic energy and thermal energy are far smaller than the turbulent energy $\left(1.6 \times 10^{48} \mathrm{erg}\right)$ of filament G47.06+0.26. Compared to the kinetic energy and thermal energy, the ionization energy $\left(3.2 \times 10^{48} \mathrm{erg}\right)$ may inject more energy into the $\mathrm{G} 47.06+0.26$, and could help maintain the turbulence.

4. We found nine BGPS sources within G47.06+0.26. These BGPS sources have sufficient mass to form massive stars. The obtained clump formation efficiency (CFE) is $\sim 18 \%$ in the filament $\mathrm{G} 47.06+0.26$, which is greater than the value $(\sim 11 \%)$ in the GMC of the Milky Way. We suggest that the feedback from H II regions may be responsible for the higher $\mathrm{CFE}$. The feedback from the H II regions may cause the formation of a new generation of stars and the higher CFE in filament G47.06+0.26. The selected class I YSOs are clustered along G47.06+0.26. Comparing the ages of the two H II regions associated with N98 and B1 with that of class I YSOs $\left(\sim 10^{5} \mathrm{yr}\right)$, we infer that the expansion of the H II regions may trigger the formation of a new generation of stars, including what is now seen as class I YSOs in filament G47.06+0.26.

Acknowledgements. We are very grateful to the anonymous referee for his/her helpful comments and suggestions. We are grateful to the staff at the Qinghai Station of PMO for their assistance during the observations. Thanks for the Key Laboratory for Radio Astronomy, CAS, for partly supporting the telescope operation. This work has made use of data from the Spitzer Space Telescope, which is operated by the Jet Propulsion Laboratory, California Institute of Technology under a contract with NASA. The ATLASGAL project is a collaboration between the Max-Planck-Gesellschaft, the European Southern Observatory (ESO) and the Universidad de Chile. It includes projects E-181.C-0885 E-078.F-9040(A), M-079.C-9501(A), M-081.C-9501(A) plus Chilean data. This work was supported by the National Natural Science Foundation of China (Grant Nos. 11363004, 11403042, and 11673066).

\section{References}

Allen, L. E., Calvet, N., \& D’Alessio, P. 2004, ApJS, 154, 363

Anderson, L. D., Bania, T. M., Balser, D. S., \& Rood, R. T. 2011, A\&A, 529, L6 André, P., \& Montmerle, T. 1994, ApJ, 420, 837

André, P., Di Francesco, J., Ward-Thompson, D., et al. 2014, Protostars and Planets VI, 27

Arce, H. G., Borkin, M. A., Goodman, A. A., Pineda, J. E., \& Beaumont, C. N. 2011, ApJ, 742, 105
Bally, J., Aguirre, J., Battersby, C., et al. 2010, ApJ, 721, 137 Banerjee, R., \& Pudritz, R. E. 2008, PASP, 387, 216 Battisti, A. J., \& Heyer, M. H. 2014, ApJ, 780, 173 Benjamin, R. A., Churchwell, E., \& Babler, B. L. 2003, PASP, 115, 953 Busquet, G., Zhang, Q., Palau, A., et al. 2013, ApJ, 764, 26 Carey, S. J., Noriega-Crespo, A., Mizuno, D. R., et al. 2009, PASP, 121, 76 Castets, A., Langer, W. D., \& Wilson, R. W. 1982, ApJ, 262, 590 Churchwell, E., Povich, M. S., Allen, D., et al. 2006, ApJ, 649, 759 Churchwell, E., Watson, D. F., Povich, M. S., et al. 2007, ApJ, 670, 428 Condon, J. J. 1992, ARA\&A, 30, 575

Condon, J. J., Cotton, W. D., Greisen, E. W., et al. 1998, AJ, 115, 1693 Contreras, Y., Aray, G., Rathborne, J. M., \& Sanhueza, P. 2016, MNRAS, 456, 2041

Deharveng, L., Zavagno, A., Schuller, F., et al. 2009, A\&A, 496, 177 Dewangan, L. K., Ojha, D. K., Luna, A., et al. 2016, ApJ, 819, 66

Dunham, M. K., Rosolowsky, E., Evans, Neal, J. II., et al. 2010, ApJ, 717, 1157 Dyson, J. E., \& Williams, D. A. 1980, Physics of the interstellar medium, eds. J. E. Dyson, \& D. A. Williams (New York: Halsted Press)

Eden, D. J., Moore, T. J. T., Plume, R., \& Morgan, L. K. 2012, MNRAS, 422, 3178

Eden, D. J., Moore, T. J. T., Morgan, L. K., et al. 2013, MNRAS, 431, 1587 Elmegreen, B. G. 1998, in ASP Conf. Ser. 148, eds. C. E. Woodward, J. M. Shull, \& H. A. Tronson, 150

Enoch, M. L., Young, K. E., Glenn, J., et al. 2006, ApJ, 638, 293

Fernández-López, M., Arce, H. G., Looney, L., et al. 2014, ApJ, 790, L19

Foster, J. B., Arce, H. G., Kassis, M., et al. 2014, ApJ, 791, 108

Franco, J. 1983, ApJ, 264, 508

Freyer, T., Hensler, G., \& Yorke, H. W. 2003, ApJ, 594, 888

Garden, R. P., Hayashi, M., Hasegawa, T., et al. 1991, ApJ, 374, 540

Ginsburg, A., Glenn, J., Rosolowsky, E., et al. 2013, ApJS, 208, 14

Inutsuka, S.-I., \& Miyama, S. M. 1992, ApJ, 388, 392

Jackson, J. M., Finn, S. C., Chambers, E. T., et al. 2010, ApJ, 719, L185

Kauffmann, J., \& Pillai, T. 2010, ApJ, 723, L7

Kirk, H., Myers, P. C., Bourke, T. L., et al. 2013, ApJ, 766, 115

Lada, C. J., \& Lada, E. A. 2003, ARA\&A, 154, 363

Lasker, B. M. 1967, ApJ, 149, 23

Leger, A., \& Puget, J. L. 1984, A\&A, 137, 5

Liu, T., Zhang, Q., Kim, K.-T., et al. 2016, ApJ, 222, 7

Molinari, S., Swinyard, B., Bally, J., et al. 2010, PASP, 122, 314

Moore, T. J. T., Bretherton, D. E., Fujiyoshi, T., et al. 2007, MNRAS, 379, 663

Myers, P. C. 2009, ApJ, 700, 1609

Nakamura, F., Hanawa, T., \& Nakano, T. 1993, PASP, 45, 551

Narayanan, G., Heyer, M. H., Brunt, C., et al. 2008, ApJS, 177, 341

Norman, C., \& Silk, J. 1980, ApJ, 238, 158

Peretto, N., Fuller, G. A., Duarte-Cabral, A., et al. 2013, A\&A, 555, A112

Polychroni, D., Schisano, E., Elia, D., et al. 2013, ApJ, 777, L33

Pomarès, M., Zavagno, A., Deharveng, L., et al. 2014, A\&A, 496, A177

Rosolowsky, E., Dunham, M. K., Ginsburg, A., et al. 2010, ApJS, 188, 123

Schuller, F., Menten, K. M., Contreras, Y., et al. 2009, A\&A, 504, 415

Shu, Frank, H., Adams, Fred C., \& Lizano, S. 1987, ARA\&A, 25, 23

Simpson, R. J., Povich, M. S., Kendrew, S., et al. 2012, MNRAS, 424, 2442

Smith, R. J., Glover, S. C. O., Bonnell, I. A., et al. 2011, MNRAS, 411, 1354

Smith, R. J., Glover, S. C. O., Klessen, R. S., \& Fuller, G. A. 2016, MNRAS, 455,3640

Tackenberg, J., Beuther, H., Henning, T., et al. 2014, A\&A, 565, A101 Tomisaka, K. 1995, ApJ, 438, 226

Wang, K., Testi, L., Ginsburg, A., et al. 2015, MNRAS, 450, 4043

Xi, H., Zhou, J. J., Esimbek, J., et al. 2015, MNRAS, 453, 4203

Xu, J.-L., Li, D., Zhang, C.-P., et al. 2016, ApJ, 819, 117

Zhang, Q., Wang, K., Lu, X., \& Jiménez-Serra, I. 2015, ApJ, 804, 141 